\title{
A fully probe corrected near-field far-field transformation technique employing plane-wave synthesis
}

\author{
R. A. M. Mauermayer and T. F. Eibert \\ Lehrstuhl für Hochfrequenztechnik, Technische Universität München, 80290 Munich, Germany \\ Correspondence to: R. A. M. Mauermayer (raimund.mauermayer@tum.de)
}

\begin{abstract}
The far-field behavior of an antenna under test (AUT) can be obtained by exciting the AUT with a plane wave. In a measurement, it is sufficient if the plane wave is artificially generated in the vicinity of the AUT. This can be achieved by using a virtual antenna array formed by a probe antenna which is sequentially sampling the radiating nearfield of the AUT at different positions. For this purpose, an optimal filter for the virtual antenna array is computed in a preprocessing step. Applying this filter to the near-field measurements, the far-field of the AUT is obtained according to the propagation direction and polarization of the synthesized plane wave. This means that the near-field far-field transformation (NFFFT) is achieved simply by filtering the near-field measurement data. Taking the radiation characteristic of the probe antenna into account during the synthesis process, its influence on the NFFFT is compensated.

The principle of the plane-wave synthesis and its application to the NFFFT is presented in detail in this paper. Furthermore, the method is verified by performing transformations of simulated near-field measurement data and of near-field data measured in an anechoic chamber.
\end{abstract}

\section{Introduction}

Today's wireless communication, radar or direction finding systems make use of electrically large antennas like parabolic reflectors or antenna arrays to generate far-field radiation characteristics suitable for the particular application. After fabrication the far-field of the antenna under test has to be examined by measurements to verify that it meets the requirements on phase and magnitude. Due to the large size of the antenna relative to wavelength, an accurate measurement under far-field conditions inside an anechoic chamber can hardly be accomplished, simply because of the enormous measurement distance required. Thus, performing measure- ments in a reduced distance in the radiating near-field region of the AUT and subsequently applying a near-field far-field (NFFF) transformation is an attractive alternative.

The radiated fields of the AUT and the probe are usually represented by a truncated series of orthogonal spherical, cylindrical or planar field modes to formulate a transmission equation describing the coupling between both antennas (Hansen, 1988). Solving this equation for the modal coefficients the far-field can be computed by evaluating the field modes for the radial distance going to infinity.

For the plane-wave synthesis approach (Hansen, 1988; Yamaguchi et al., 2009; Bennett and Schoessow, 1978), there is no explicit transmission equation needed. The probe which is sampling the near-field successively at different locations is considered to form a virtual array of probe antennas on a measurement surface. This array is then used to synthesize a plane wave in the vicinity of the AUT through a weighted superposition of the fields radiated by the elements of the virtual probe antenna array. The appropriate weights form a filter which is gained from the solution of an inverse problem.

The proposed method allows shifting most of the computational expense of the NFFF transformation and probe correction to a preprocessing step whose result is a set of filters containing the weighting factors for the probe signals. These filters are used to compute the far-field directly from the acquired measurement data in a near-field far-field transformation step.

The paper is structured as follows: in the following part the principle and the results of the plane-wave synthesis is shown. How the synthesis is employed for near-field far-field transformation is given in the third part. In the forth part, the method is applied to simulated and real near-field measurement data of a Yagi-Uda and a patch antenna. The results of the transformation together with some accuracy considerations are presented. 


\section{The probe corrected plane-wave synthesis}

\subsection{Principle}

The principle of the synthesis of plane waves by a virtual array of probes which are positioned at different locations in the near-field of the AUT is visualized in Fig. 1. The probes are excited according to the filter vector $\boldsymbol{w}\left(\hat{k}, \boldsymbol{E}_{0}\right)$, which consists of $N$ complex coefficients, one for each probe position and polarization orientation. The objective is to generate the field of the plane wave

$$
\begin{aligned}
& \boldsymbol{E}_{\mathrm{pl}}(\boldsymbol{r})=\boldsymbol{E}_{0} e^{-j k \hat{k} \cdot \boldsymbol{r}} \\
& \boldsymbol{H}_{\mathrm{pl}}(\boldsymbol{r})=\frac{1}{Z_{0}} \hat{k} \times \boldsymbol{E}_{\mathrm{pl}}(\boldsymbol{r})
\end{aligned}
$$

in the vicinity of the AUT by superimposing the probe fields. The propagation direction of the plane wave is described by the unit vector $\hat{k}$ and its polarization is given by the electric field vector $\boldsymbol{E}_{0} . Z_{0}$ describes the free space wave impedance.

The virtual array is equivalent to a probe antenna with ideal polarization purity positioned in the far-field of the AUT in direction $-\hat{k}$ and exciting the AUT with a quasi plane-wave field with the same polarization as the synthesized plane wave. The power wave $b_{1}^{\mathrm{FF}}\left(\hat{k}, \boldsymbol{E}_{0}\right)$ at the feed point of the AUT in Fig. 1 is proportional to this far-field measurement.

For the wave field synthesis process inside a volume, the AUT is enclosed by a Huygens' surface. A filter must be found that enables the synthesis of the tangential field components of the desired plane wave on the surface. The uniqueness theorem (Harrington, 2001) guarantees that if the superimposed tangential field components of the virtual probe array correspond to those of the plane wave, the plane-wave field is also present inside the source free test volume.

The probe antenna is modeled by its equivalent electric $\boldsymbol{J}_{n}\left(\boldsymbol{r}^{\prime}\right)$ and magnetic $\boldsymbol{M}_{n}\left(\boldsymbol{r}^{\prime}\right)$ surface currents located at the $n$-th probe sampling position. Its characteristic electromagnetic field is computed from the surface integrals

$$
\begin{aligned}
\boldsymbol{E}_{n}(\boldsymbol{r}) & =\iint_{A}\left[\overline{\mathbf{G}}_{J}^{E}\left(\boldsymbol{r}, \boldsymbol{r}^{\prime}\right) \cdot \boldsymbol{J}_{n}\left(\boldsymbol{r}^{\prime}\right)\right. \\
& \left.+\overline{\mathbf{G}}_{M}^{E}\left(\boldsymbol{r}, \boldsymbol{r}^{\prime}\right) \cdot \boldsymbol{M}_{n}\left(\boldsymbol{r}^{\prime}\right)\right] d A^{\prime} \\
\boldsymbol{H}_{n}(\boldsymbol{r}) & =\iint_{A}\left[\overline{\mathbf{G}}_{J}^{H}\left(\boldsymbol{r}, \boldsymbol{r}^{\prime}\right) \cdot \boldsymbol{J}_{n}\left(\boldsymbol{r}^{\prime}\right)\right. \\
& \left.+\overline{\mathbf{G}}_{M}^{H}\left(\boldsymbol{r}, \boldsymbol{r}^{\prime}\right) \cdot \boldsymbol{M}_{n}\left(\boldsymbol{r}^{\prime}\right)\right] d A^{\prime},
\end{aligned}
$$

where $\overline{\mathbf{G}}_{J / M}^{E / H}\left(\boldsymbol{r}, \boldsymbol{r}^{\prime}\right)$ are the dyadic Greens' functions for free space. The tangential components of the probe field are sampled at discrete locations $\boldsymbol{r}_{i}(i=1, \ldots, I)$ on the Huygens' surface. In order to avoid the excitation of resonance modes

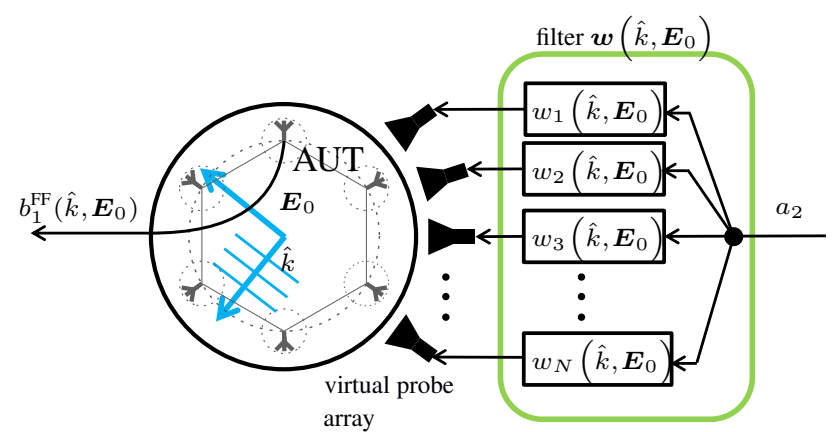

Fig. 1. The principle of plane-wave synthesis by a virtual array of probes positioned in the near-field region of the AUT. The AUT consists of six antenna elements. The power wave $b_{1}^{\mathrm{FF}}\left(\hat{k}, \boldsymbol{E}_{0}\right)$ is measured at the feed point of one antenna element.

inside the test volume, the combined field vector (Hansen, 1988)

$\boldsymbol{F}_{n}\left(\boldsymbol{r}_{i}\right)=\left.\boldsymbol{E}_{n}\left(\boldsymbol{r}_{i}\right)\right|_{\tan }-Z_{0} \boldsymbol{n} \times \boldsymbol{H}_{n}\left(\boldsymbol{r}_{i}\right)$

is used, with $\boldsymbol{n}$ being the normal vector of the Huygens' surface. The constraint that the tangential components of the superimposed probe fields have to be equal to that of the plane wave leads to the equation system

$$
\underbrace{\left(\begin{array}{ccc}
\boldsymbol{F}_{1}\left(\boldsymbol{r}_{1}\right) & \ldots & \boldsymbol{F}_{N}\left(\boldsymbol{r}_{1}\right) \\
\boldsymbol{F}_{1}\left(\boldsymbol{r}_{2}\right) & \ldots & \boldsymbol{F}_{N}\left(\boldsymbol{r}_{2}\right) \\
\vdots & \ddots & \vdots \\
\boldsymbol{F}_{1}\left(\boldsymbol{r}_{I}\right) & \ldots & \boldsymbol{F}_{N}\left(\boldsymbol{r}_{I}\right)
\end{array}\right)}_{\mathbf{F}} \underbrace{\left(\begin{array}{c}
w_{1}\left(\hat{k}, \boldsymbol{E}_{0}\right) \\
\vdots \\
w_{N}\left(\hat{k}, \boldsymbol{E}_{0}\right)
\end{array}\right)}_{\boldsymbol{w}\left(\hat{k}, \boldsymbol{E}_{0}\right)}=\underbrace{\left(\begin{array}{c}
\boldsymbol{F}_{\mathrm{pl}}\left(\boldsymbol{r}_{1}\right) \\
\vdots \\
\boldsymbol{F}_{\mathrm{pl}}\left(\boldsymbol{r}_{I}\right)
\end{array}\right)}_{\boldsymbol{F}_{\mathrm{pl}}}
$$

of the inverse problem with $\boldsymbol{F}_{\mathrm{pl}}\left(\boldsymbol{r}_{i}\right)$ being the combined field vector evaluated for the plane wave. As there are usually more sampling points $I$ on the Huygens' surface than probe positions $N$, the equation system is over-determined. Therefore, it is solved for the filter coefficients in a least mean square error sense in form of

$\boldsymbol{w}\left(\hat{k}, \boldsymbol{E}_{0}\right)=\mathbf{F}^{+} \boldsymbol{F}_{\mathrm{pl}}$

using the pseudoinverse $\mathbf{F}^{+}$of matrix $\mathbf{F}$ in Eq. (6). This solution also minimizes the norm of the filter vector and the power radiated by the virtual probe antenna array, respectively.

In order to avoid 3-D measurements, it is also investigated whether it is possible to synthesize a plane-wave field in a flat region around the AUT using a virtual array of probe antennas positioned on a closed curve around the test region. The motivation for this idea is to improve the accuracy of far-field measurement results for an antenna measurement facility that only allows to record near-field data for some great circles but not on a complete measurement surface. 
For this synthesis problem, the field components of the probe are directly sampled in the flat area instead of sampling the tangential field components on a Huygens' surface. However the kind of inverse problem remains the same and it is solved in the same way as described above.

\subsection{Measurement setups and sampling conditions}

The measurement setup has to be defined for the synthesis process. There are two setups for the investigation of the plane-wave synthesis inside a volume and on a planar area.

For the synthesis in a volume the spherical measurement setup depicted in Fig. $2 \mathrm{a}$ is considered. The probe is positioned on a spherical measurement surface with radius $1 \mathrm{~m}$. At each position of the regular sampling grid in $\vartheta$ and $\varphi$ direction the probe is oriented with vertical and horizontal polarization. The probe sampling rate, which depends on wavelength $\lambda$ and the radius $\rho$ of the minimum sphere, is derived from the sampling condition (Yaghjian, 1986)

$$
\Delta \vartheta=\Delta \varphi \leq \frac{\lambda}{2(\rho+\lambda)}
$$

for conventional NFFFT using spherical multipoles. The Huygens' surface is formed by the minimum sphere which encloses the AUT and has a radius of $\rho=0.5 \mathrm{~m}$.

For the synthesis of a plane wave on a planar area the setup shown in Fig. $2 b$ is used. The intention here is to generate a field distribution on the disc with radius $0.5 \mathrm{~m}$ that is more similar to a plane wave than the field of the probe antenna itself. The probe is positioned on a measurement circle in the $x y$ plane with radius $1.98 \mathrm{~m}$. Again the probe sampling interval in $\varphi$ direction is chosen following Eq. (8).

The sampling point distance of the probe fields on the Huygens' surface and on the disc is chosen to be smaller than $\lambda / 2$ according to the Nyquist-Shannon sampling theorem.

\subsection{Filter coefficients}

For the spherical measurement, an open ended rectangular waveguide probe operating at $2 \mathrm{GHz}$ is used. It is positioned on the measurement surface in $5^{\circ}$ steps in $\vartheta$ and $\varphi$ direction. The tangential field components on the Huygens' surface are also sampled on a regular grid in $\vartheta$ and $\varphi$ direction in $6^{\circ}$ intervals. For all probe positions the probe fields are computed on the Huygens' surface by Eq. (3) and Eq. (4). The sampled tangential components result in the columns of matrix $\mathbf{F}$ in Eq. (6). The plane wave that is to be synthesized is propagating in positive $x$ direction and is vertically polarized as it is given in Fig. 2a. The necessary filter coefficients for the optimal synthesis of the plane wave are gained from the solution of the equation system according to Eq. (7). In Fig. 3 the resulting normalized magnitude of the filter coefficients is shown. As the probe is vertically and horizontally oriented there is one filter coefficient for each of the probe polarization states. It can be clearly seen that most of the power is

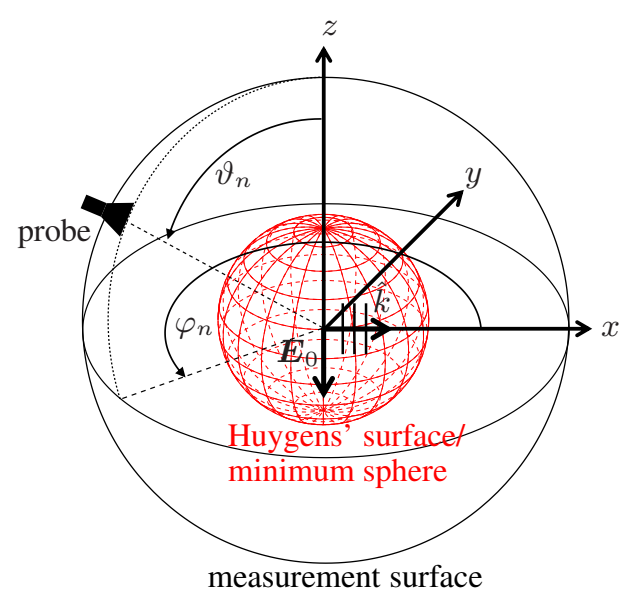

(a) Spherical measurement setup. A plane wave is synthesized inside the minimum sphere enclosing the AUT with propagation direction $\hat{k}=$ $\boldsymbol{e}_{x}$ and vertical polarization $\boldsymbol{E}_{0}=-\boldsymbol{e}_{z}$

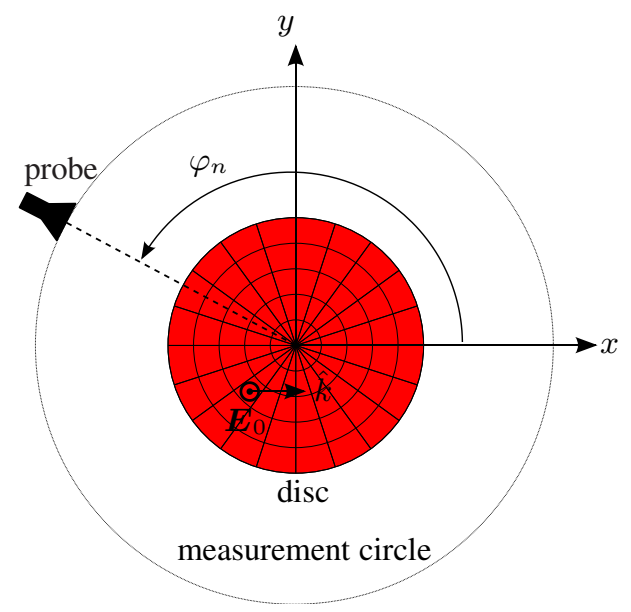

(b) Measurement setup for plane-wave synthesis on a disc. The probe is rotated on the measurement circle.

Fig. 2. Measurement setups for plane-wave synthesis inside a volume (a) and on a disc (b).

radiated by the vertically polarized probes which are radiating in propagation direction of the plane wave whereas the horizontally oriented probes are excited with less power because their radiated fields have a dominant horizontal electric field component and fit less to the vertically polarized plane wave.

A horn antenna probe, also operating at $2 \mathrm{GHz}$, is used for the synthesis on a disc. It is rotated on the measurement circle in $5^{\circ}$ steps. Due to the high polarization purity of the probe field on the disc, it is only oriented in vertical polarization to synthesize a vertically polarized plane wave propagating in positive $x$ direction. Figure 4 shows the normalized magnitude of the resulting filter coefficients. As for the spherical 


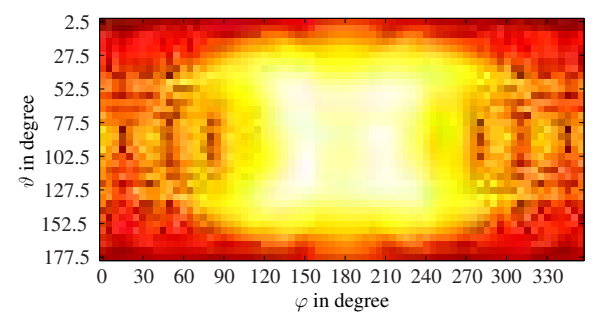

(a) vertical polarization

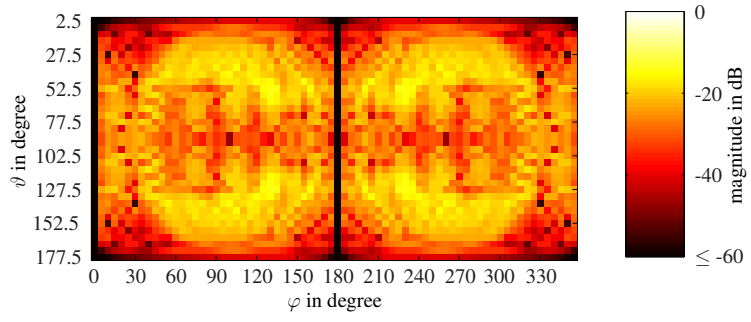

(b) horizontal polarization

Fig. 3. Normalized magnitude of the filter coefficients for a vertically (a) and a horizontally (b) polarized open ended waveguide probe synthesizing a vertically polarized plane wave propagating in positive $x$ direction.

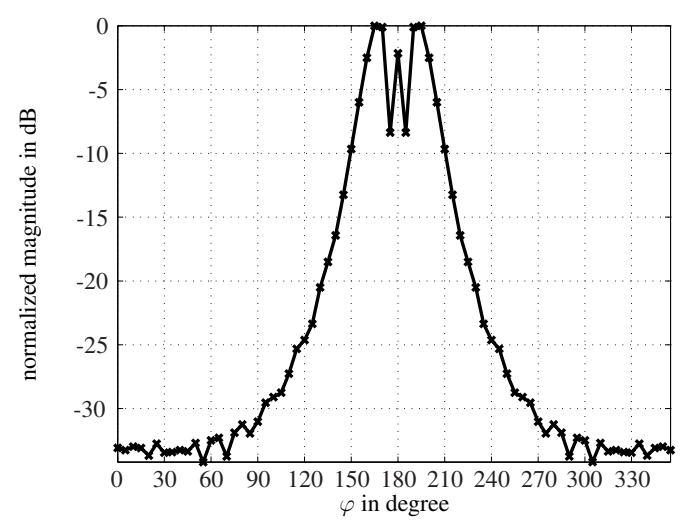

Fig. 4. Normalized magnitude of the filter coefficients for the synthesis on a disc.

setup, most of the power is radiated by the elements of the virtual probe antenna array which are already pointing in the propagation direction of the plane wave, i.e. probes on the measurement circle with $\varphi_{n}$ around $180^{\circ}$.

\subsection{Wave field synthesis results}

The elements of the virtual probe array are excited according to the computed filter coefficients. The most important accuracy measure of the synthesis method is the quality of the wave field which is generated. Since the quality of the plane wave determines the accuracy of the following NFFF transformation process. Therefore, the deviation of the magnitude and phase of the synthesized wave field from the ideal plane wave is regarded.

In the case of the spherical setup, the errors are computed on lines which are crossing the minimum sphere in different directions to get an impression of the electrical field distribution inside the volume. The deviations are shown in Fig. 5. The magnitude error of the generated wave field is lower than $-80 \mathrm{~dB}$. The phase error is smaller than $0.006^{\circ}$. As only the $z$ component of the synthesized electric field is considered, the polarization purity was checked to be above $80 \mathrm{~dB}$ inside the minimum sphere. It is possible to further increase the qual- ity of the synthesized plane wave by increasing the sampling rate of the probe on the measurement surface.

The errors of the plane wave synthesized on the disc are shown in Fig. 6. The magnitude shows a maximum deviation of about $-17 \mathrm{~dB}$ and the phase of about $2^{\circ}$. It should be mentioned that the errors increase below and above the considered planar region in $z$ direction. Nevertheless, as it was intended, the superimposed field on the disc of the virtual array is more similar to a plane wave than the field of only one probe on the measurement circle, which would result in a maximum deviation of $-11 \mathrm{~dB}$ in magnitude and $140^{\circ}$ in phase.

In general, a closed boundary surface with the appropriate impressed equivalent surface currents is mandatory for the accurate generation of a desired wave field. For the synthesis of the plane wave on a disc, this assumption is violated since the virtual probe array only forms kind of a line current around the disc. Therefore an accurate synthesis of the desired wave field cannot be achieved.

\section{NFFFT based on plane-wave synthesis}

To find a relation between the near-field measurements and the AUT far-field, the signal flow in Fig. 1 is inverted so that the transposed system shown in Fig. 7 is obtained. This can be done due to the assumption of the reciprocity property of the whole system. The far-field of the AUT in direction $-\hat{k}$, which is now proportional to the power wave signal $b_{2}^{F F}\left(\hat{k}, \boldsymbol{E}_{0}\right)$, is computed by

$b_{2}^{F F}\left(\hat{k}, \boldsymbol{E}_{0}\right)=\sum_{n=1}^{N} w_{n}\left(\hat{k}, \boldsymbol{E}_{0}\right) b_{2, n}^{N F}$

applying the filter coefficients $w_{n}\left(\hat{k}, \boldsymbol{E}_{0}\right)$ to the power wave signals $b_{2, n}^{\mathrm{NF}}$ received by the probe at position $n$ in the AUT near-field. From Eq. (9) it is obvious that the NFFF transformation employing plane-wave synthesis is just a filtering of the near-field data.

Although the filter is computed for only one specific direction $\hat{k}$, the propagation direction of the plane wave can be 


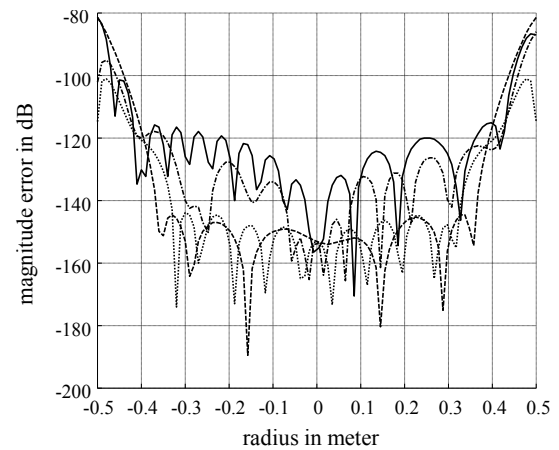

(a) magnitude error
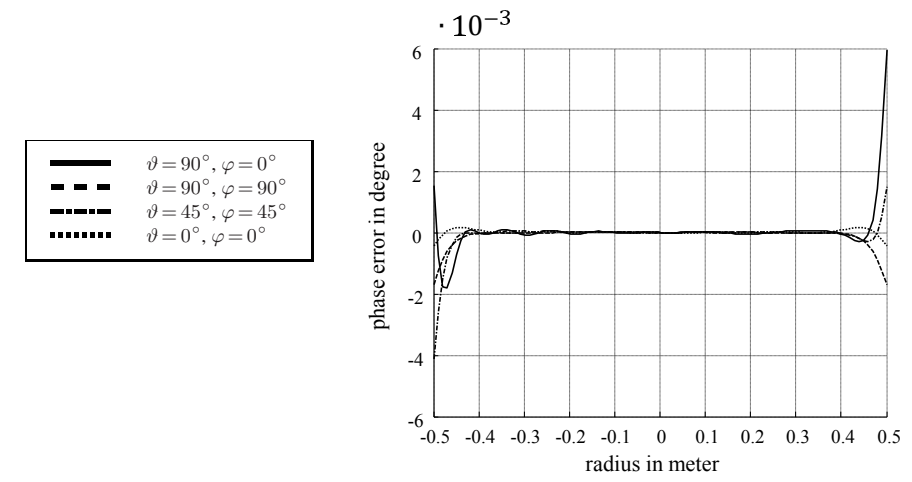

(b) phase error

Fig. 5. Magnitude and phase error of the synthesized plane wave evaluated on several lines crossing the minimum sphere in different directions.

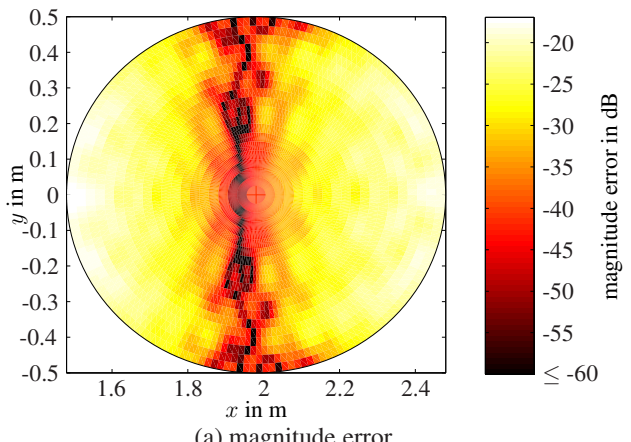

(a) magnitude error

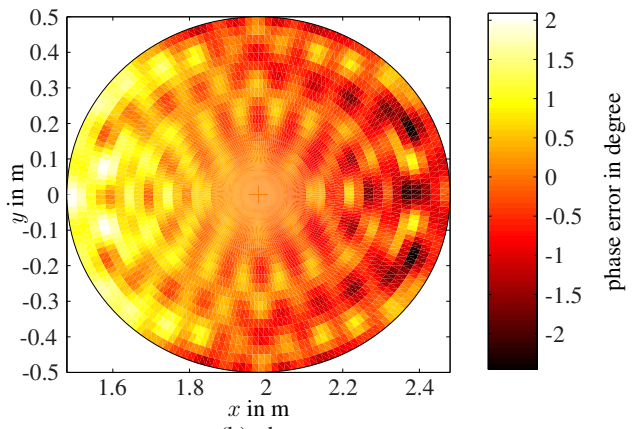

(b) phase error

Fig. 6. Magnitude and phase error of the synthesized plane wave on a disc.

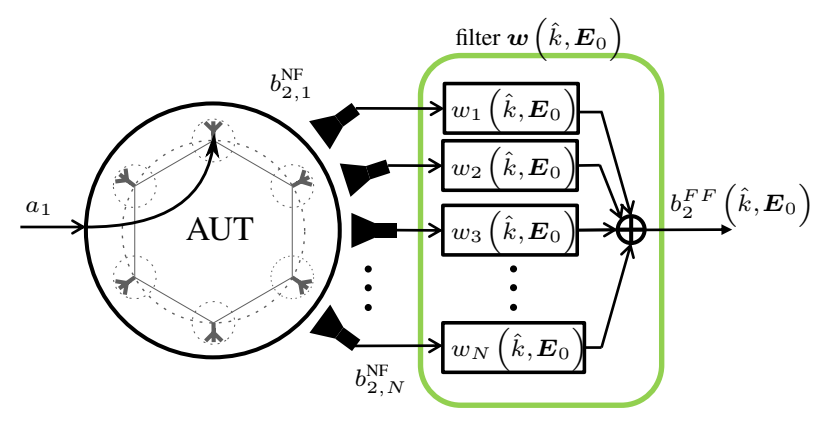

Fig. 7. Considered system for the NFFFT employing plane-wave synthesis.

rotated in $\varphi$ direction by a cyclic rotation of the filter coefficients taking advantage of the rotational symmetry of the probe sampling points on the measurement and of the Huygens' surface. This allows to determine a horizontal cut of the AUT far-field pattern (E-plane) without having to solve the inverse problem of Eq. (6) for each $\varphi$ angle of plane wave incidence again.

\section{Application of the NFFFT based on plane-wave synthesis}

\subsection{Simulated and measured near-field data}

A spherical near-field measurement is simulated with FEKO (EM Software and Systems, 2011) for the verification of the proposed method of synthesizing a plane wave inside a volume. A three element Yagi-Uda antenna composed of wire elements is used as AUT. It is vertically polarized and its main beam direction is pointing in positive $x$ direction. The simple structure of the antenna makes a low computation time possible needed for the simulation of about 5000 nearfield measurement points. Figure 8 shows the normalized magnitude of the received probe signal for copolar orientation of the waveguide probe. 


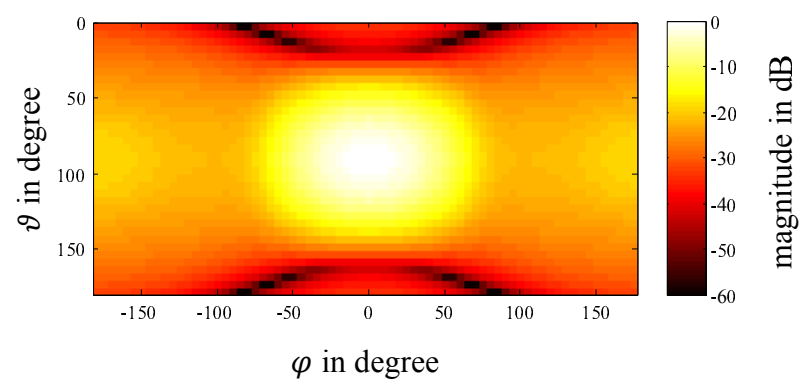

Fig. 8. Normalized magnitude of the probe signal for copolar orientation of the waveguide probe.

The NFFFT based on the wave field synthesis on a disc is verified applying it to simulated near-field data acquired by the horn antenna probe in the near-field of a patch antenna. This simulation scenario is also built up in an anechoic chamber, shown in Fig. 10, and real measurements are recorded. Since the AUT represents one element of a circular antenna array it is mounted to a construction made from Rohacell so that the patch antenna pattern is not disturbed by the mounting. The normalized, simulated and measured probe signals are plotted in Fig. 9.

Both AUT antennas are positioned in $0.3 \mathrm{~m}$ distance to the rotational axis as they represent the antenna element of a circular antenna array which is sketched in Fig. 1.

The simulated and real measurement setups, including the probe and sampling rates, are the same as for the synthesis processes, so the computed filters can be directly applied to the simulated and measured near-field data for NFFF transformation.

\subsection{NFFF transformation results}

For the spherical NFFF transformation the filter of Fig. 3 is directly applied to the acquired near-field data of Fig. 8. Evaluating Eq. (9) for the cyclically rotated versions of the filter coefficients leads to the horizontal cut (H-plane) of the farfield pattern. The transformation result compared to the reference solution computed by FEKO is given in Fig. 11. As it is to be expected from the high quality of the synthesized plane wave in the vicinity of the AUT there is no difference recognizable between reference and NFFFT results. Regarding the errors of the transformation in Fig. 12, it can be seen that the error of the normalized far-field magnitude is below $-80 \mathrm{~dB}$ and the phase error is in the range of some thousandths of one degree. So the transformation errors are in the range of the magnitude and phase errors of the synthesized plane wave.

Already from the quality of the synthesized plane-wave field on the disc it can be derived that the transformation results of the near-field data acquired on the measurement circle cannot reach the accuracy as the one of the complete spherical measurement. Figure 13 shows the results of the

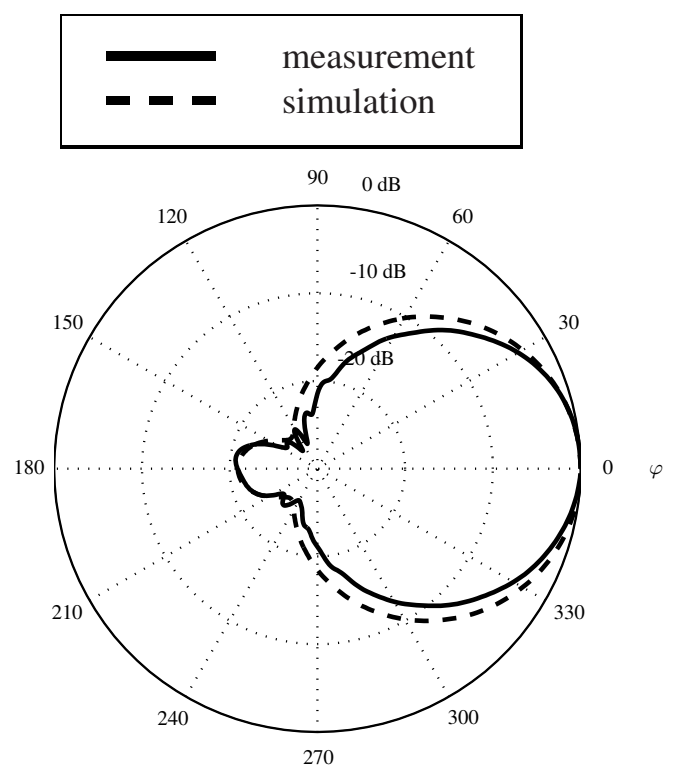

Fig. 9. Simulated and measured near-field data acquired on the measurement circle.

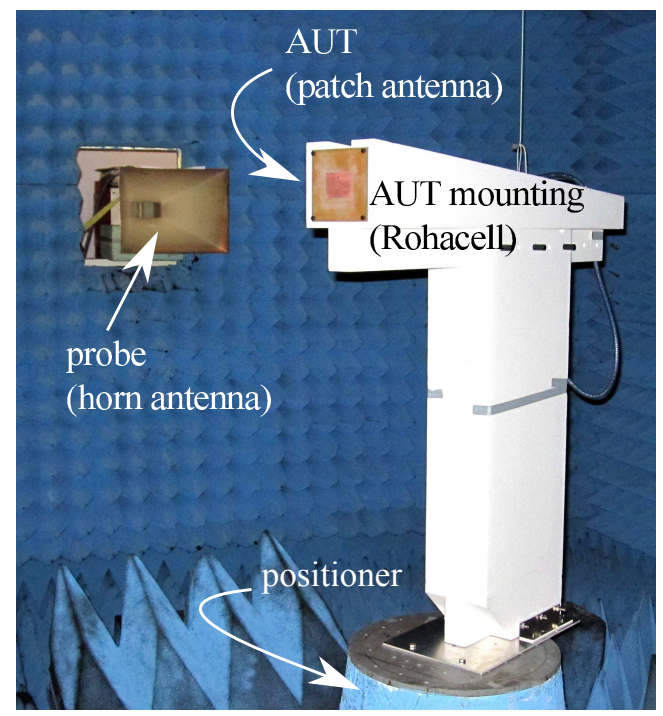

Fig. 10. Measurement setup in an anechoic chamber for the NFFFT based on the plane-wave synthesis on a disc.

NFFF transformation for the simulated and measured nearfield data. For the simulated data, the transformation result matches very well to the reference of the patch antenna farfield pattern.

The positive effect of the transformation is also visible for the measured near-field data. However, there is still a major deviation from the reference compared to the transformation results of the simulated near-field data. The main reason for 

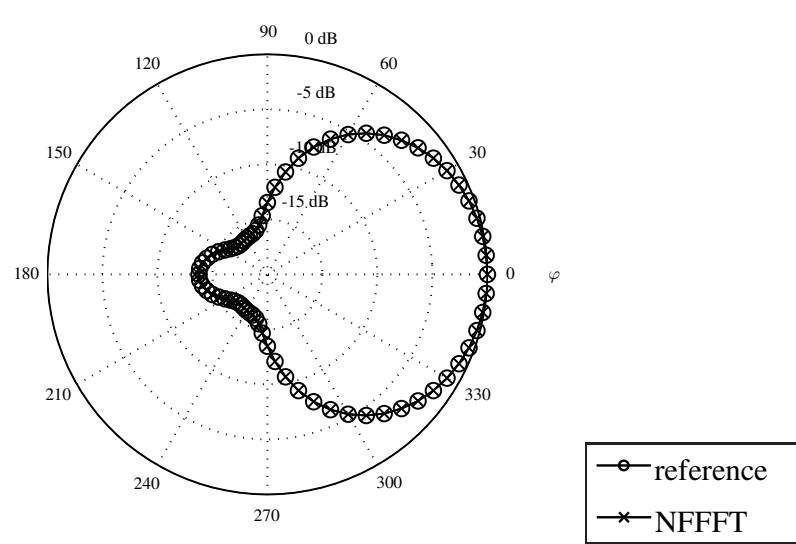

Fig. 11. Magnitude of reference and transformed far-field.

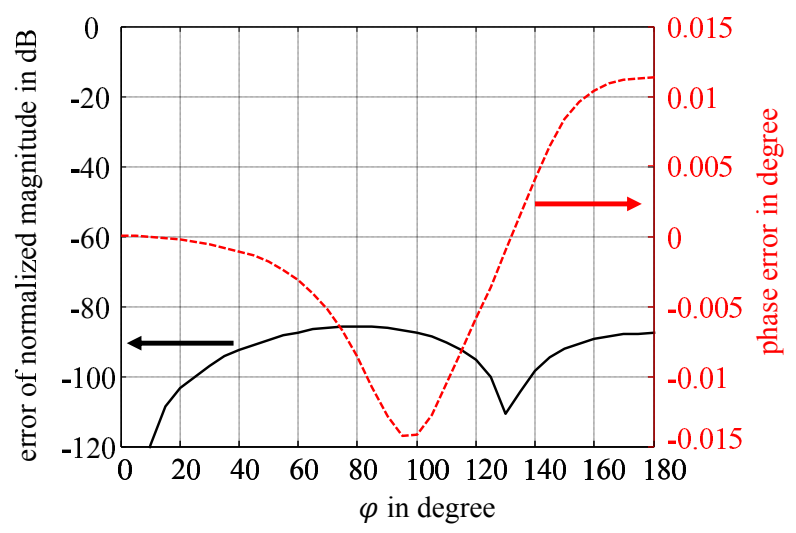

Fig. 12. Errors of normalized magnitude and phase of the transformation result for the spherical near-field measurement.

this seems to be that the measurement was done in a facility that is not appropriate for near-field measurements, so that the exact positioning and orientation of AUT and probe antennas could not be guaranteed. This means that the probe field on the disc which was assumed for the synthesis might have been different from the probe field during the measurement in the anechoic chamber. This also explains the difference between simulated and measured near-field data in Fig. 9.

\section{Conclusions}

The presented near-field far-field transformation technique based on plane-wave synthesis allows to split the transformation process into two steps. In the first step, the plane wave is synthesized by solving an inverse problem for the filter vector for the virtual probe array, which might be time consuming. In the second step, the transformation of the nearfield data can be performed by a faster filtering procedure.

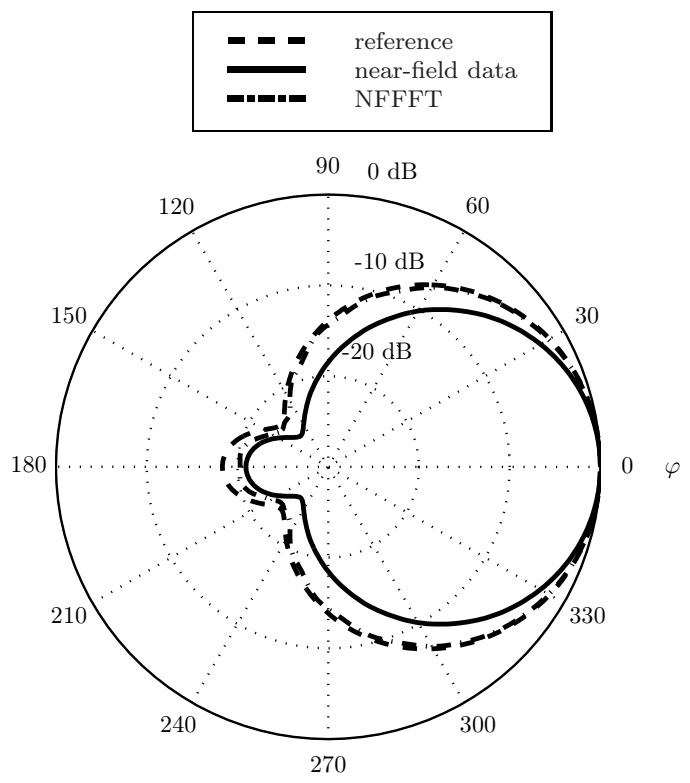

(a) simulation

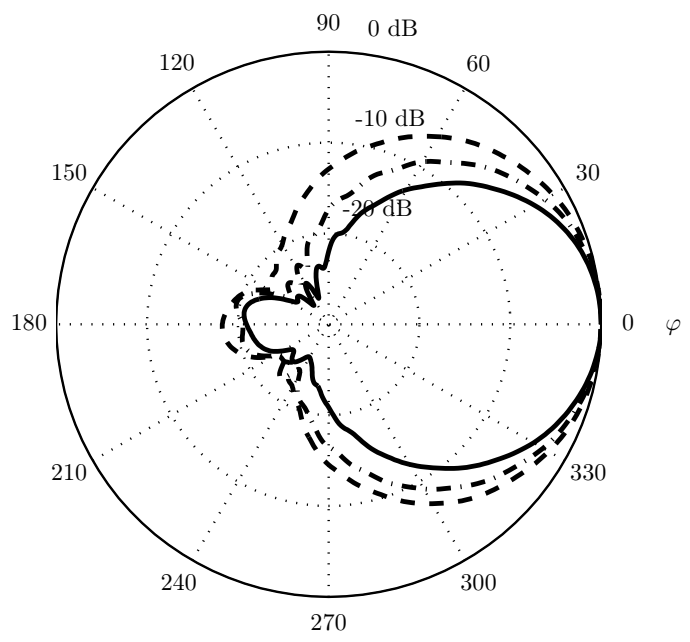

(b) measurement

Fig. 13. Results of the NFFF transformation of the simulated and measured near-field data acquired on the measurement circle.

The method was verified by applying it to simulated and real near-field measurement data.

If the computed filters are stored they can be reused for the transformation of near-field measurement data of different AUTs as long as the measurement setup including probe, probe sampling rate, frequency, measurement surface and the shape of the Huygens' surface do not change. The synthesis of arbitrary field distributions inside arbitrarily shaped volumes is an issue of further investigation. 
54 R. A. M. Mauermayer and T. F. Eibert: A fully probe corrected NFFFT technique employing plane-wave synthesis

\section{References}

Bennett, J. and Schoessow, E.: Antenna near-field/far-field transformation using a plane-wave-synthesis technique, Proceedings of the Institution of Electrical Engineers, 125, 179-184, doi:10.1049/piee.1978.0048, 1978.

EM Software and Systems: FEKO Suite 6.1, http://www.feko.info, 2011.

Hansen, J. E.: Spherical near-field antenna measurements, IEE Electromagnetic Waves Series 26, Peter Peregrinus Ltd., London, UK, 1988.
Harrington, R. F.: Time-Harmonic Electromagnetic Fields, Wiley, J., Weinheim, 2001.

Yaghjian, A.: An overview of near-field antenna measurements, IEEE Transactions on Antennas and Propagation, 34, 30-45, doi:10.1109/TAP.1986.1143727, 1986.

Yamaguchi, R., Kimura, Y., Komiya, K., and Cho, K.: A far-field measurement method for large size antenna by using synthetic aperture antenna, in: 3rd European Conference on Antennas and Propagation, 1730-1733, 2009. 